\title{
Possible involvement of loss of imprinting in immortalization of human fibroblasts
}

\author{
KOTARO OKAMURA $^{1}$, MAKI OHNO ${ }^{1,2}$ and TAKEKI TSUTSUI ${ }^{1}$ \\ ${ }^{1}$ Department of Pharmacology, School of Life Dentistry at Tokyo, The Nippon Dental University, Tokyo 102-8159, Japan
}

Received November 8, 2010; Accepted December 30, 2010

DOI: $10.3892 /$ ijo.2011.931

\begin{abstract}
Disruption of the normal pattern of parental originspecific gene expression is referred to as loss of imprinting (LOI), which is common in various cancers. To investigate a possible role of LOI in the early stage of human cell transformation, we studied LOI in 18 human fibroblast cell lines immortalized spontaneously, by viral oncogenes, by chemical or physical carcinogens, or by infection with a retrovirus vector encoding the human telomerase catalytic subunit, $h T E R T$ cDNA. LOI was observed in all the 18 immortal cell lines. The gene most commonly exhibiting LOI was NDN which displayed LOI in 15 of the 18 cell lines (83\%). The other genes exhibiting LOI at high frequencies were PEG3 (50\%), MAGE-L2 (61\%) and ZNF 127 (50\%). Expression of $N D N$ that was lost in the immortal cell lines was restored by treatment with 5-aza-2'-deoxycytidine. The ratio of histone $\mathrm{H} 3$ lysine 9 methylation to histone $\mathrm{H} 3$ lysine 4 methylation of the chromatin containing the NDN promoter in the immortal WI-38VA13 cells was greater than that in the parental cells, suggesting chromatin structure-mediated regulation of $N D N$ expression. We previously demonstrated that inactivation of the $\mathrm{p} 16^{\mathrm{INK} 4 \mathrm{a}} / \mathrm{pRb}$ pathway is necessary for immortalization of
\end{abstract}

Correspondence to: Dr Takeki Tsutsui, Department of Pharmacology, School of Life Dentistry at Tokyo, The Nippon Dental University, 1-9-20 Fujimi, Chiyoda-ku, Tokyo 102-8159, Japan E-mail: takeki@tokyo.ndu.ac.jp

Present address: ${ }^{2}$ Faculty of Pharmacy, Teikyo Heisei University, 2289 Uruido, Ichihara-shi, Chiba 290-0193, Japan

\begin{abstract}
Abbreviations: 5-AZC, 5-aza-2'-deoxycytidine; ChIP, chromatin immunoprecipitation; H3-K4, histone H3 lysine 4; H3-K9, histone H3 lysine 9; hTERT, human telomerase catalytic subunit; IC, imprinting center; IGF2, insulin-like growth factor II; LOI, loss of imprinting; MNNG, N-methyl-N'-nitro-N-nitrosoguanidine; 4NQO, 4-nitroquinoline 1-oxide; PCR, polymerase chain reaction; $\mathrm{pRb}$, retinoblastoma protein; PWS, Prader-Willi syndrome; RT-PCR, reverse transcriptase polymerase chain reaction; SV 40, Simian virus 40; TPA, 12-O-tetradecanoyl phorbol-13-acetate
\end{abstract}

Key words: immortalization, loss of imprinting, human fibroblasts, epigenetic modification human cells. Human fibroblasts in the pre-crisis phase and cells with an extended lifespan that eventually senesce, both of which have the normal $\mathrm{p} 16^{\mathrm{INK} 4 \mathrm{a}} / \mathrm{pRb}$ pathway, did not show LOI at any imprinted gene examined. Although it is not clear if LOI plays a causal role in immortalization of human cells or is merely coincidental, these findings indicate a possible involvement of LOI in immortalization of human cells or a common mechanism involved in both processes.

\section{Introduction}

Alterations of gene expression without a change in DNA sequence, i.e. changes in gene expression mediated by epigenetic modifications, play an important role in malignant transformation of human cells (1). An imprinted gene is one of the representative genes, the expression of which is controlled by an epigenetic mechanism. Epigenetic control of imprinted genes results in expression of only one allele, which depends on the parent of origin (2). Disruption of the normal pattern of parent-of-origin-specific gene expression is referred to as loss of imprinting (LOI), which is common in various cancers (3). For example, LOI of the insulin-like growth factor II (IGF2) gene is the most common LOI event across the widest range of tumor types, including colon, liver, lung and ovarian cancer, as well as Wilms' tumor, the embryonic kidney cancer (3).

Li et al (1) examined the correlation between tumorforming ability in athymic nude mice and change in imprinted gene expression following treatment of immortal but nontumorigenic human osteoblasts with a carcinogenic agent $\mathrm{N}$-methyl-N'-nitro-N-nitrosoguanidine (MNNG) and a carcinogenic promoter 12-O-tetradecanoyl phorbol-13-acetate (TPA). They found that expression of ten imprinted genes was enhanced after the cells acquired tumorigenicity. In addition, changes in the expression of imprinted genes were accompanied by changes in the methylation status during the promoter region of the genes. The findings suggest that changes in the expression of imprinted genes mediated by an epigenetic modification can be involved in the malignant conversion of immortal human osteoblasts. Holm et al (4) also studied the correlation between LOI and cellular transformation in imprint-free mouse embryonic fibroblasts derived from imprint-free mouse embryonic stem cells. Their results indicate that LOI can predispose mouse fibroblasts to transformation, including spontaneous immortalization and subsequent tumorigenesis. It should be noted that careful 
Table I. Human immortal cell lines used in the present study.

\begin{tabular}{|c|c|c|c|c|c|}
\hline Gender & Parental cells & Immortal cells & Cell type & Agents used for immortalization & Reference \\
\hline \multirow[t]{8}{*}{ Male } & \multirow[t]{8}{*}{ WHE-7 } & $\mathrm{E} 6 \mathrm{Cl} 6$ & Fibroblast & $E 6^{\mathrm{a}}$ & (5) \\
\hline & & E7Cl 1 & Fibroblast & $E 7^{\mathrm{a}}$ & (5) \\
\hline & & $\mathrm{E} 7 \mathrm{Cl} 2$ & Fibroblast & & (5) \\
\hline & & $\mathrm{E} 7 \mathrm{Cl} 3$ & Fibroblast & & (5) \\
\hline & & $\mathrm{E} 7 \mathrm{Cl} 4$ & Fibroblast & & (5) \\
\hline & & $\mathrm{E} 6 / \mathrm{E} 7 \mathrm{Cl} 2$ & Fibroblast & $E 6 / E 7^{\mathrm{a}}$ & (5) \\
\hline & & $\mathrm{E} 6 / \mathrm{E} 7 \mathrm{Cl} 3$ & Fibroblast & & (5) \\
\hline & & $\mathrm{E} 6 / \mathrm{E} 7 \mathrm{Cl} 5$ & Fibroblast & & (5) \\
\hline \multirow[t]{5}{*}{ Male } & \multirow[t]{5}{*}{ MDAH 087} & LCS-ST & Fibroblast & Spontaneously ${ }^{\mathrm{b}}$ & (6) \\
\hline & & LCS-AF.1-2 & Fibroblast & Aflatoxin $\mathrm{B}_{1}$ & (7) \\
\hline & & LCS-AF.1-3 & Fibroblast & Aflatoxin $\mathrm{B}_{1}$ & (7) \\
\hline & & LCS-AF.3-1 & Fibroblast & Aflatoxin $\mathrm{B}_{1}$ & (7) \\
\hline & & LCS-4X2 & Fibroblast & $\mathrm{X}$-ray & (8) \\
\hline Female & WI-38 & WI-38VA13 & Fibroblast & SV $40^{c}$ & (9) \\
\hline Female & OUMS-24 & OUMS-24F & Fibroblast & $4 \mathrm{NQO}^{\mathrm{d}}$ & (10) \\
\hline Female & KMS-6 & KMST-6 & Fibroblast & ${ }^{60} \mathrm{Co}$ & (11) \\
\hline Female & Ay & Ayt & Fibroblast & $h T E R T^{\mathrm{e}}$ & (12) \\
\hline Female & Pel & Pelt & Fibroblast & hTERT & (12) \\
\hline
\end{tabular}

${ }^{a} E 6, E 7$, or $E 6 / E 7$ represents the human papilloma virus type $16 E 6, E 7$, or both $E 6$ and $E 7$, respectively; bshows that MDAH 087 cells were spontaneously immortalized; 'Simian virus 40; ${ }^{\mathrm{d}} 4$-nitroquinoline 1-oxide; ${ }^{\mathrm{e}}$ human telomerase catalytic subunit.

studies are needed when we demonstrate the causal role of LOI in the spontaneous immortalization of mouse embryonic fibroblasts, because it is well-known that mouse fibroblasts themselves have a high frequency of spontanous transformation.

The above study reported by Li et al (1) represents the possible involvement of LOI in the malignant transformation of already immortalized human cells. Studies on the effect of LOI during the immortalization process of human cells have not been performed. In the present study, to investigate a possible role of LOI in immortalization of human cells, we examined the expression of nine imprinted genes in 18 human fibroblast cell lines immortalized spontaneously, by viral oncogenes, by chemical or physical carcinogens, or by infection with a retrovirus vector encoding the human telomerase catalytic subunit, hTERT cDNA, and compared with the expression in their seven parental cells.

\section{Materials and methods}

Cells and culture conditions. Eighteen human immortal fibroblast cell lines and their seven parental cells that we used in the present study are shown in Table I. They were immortalized spontaneously, by viral oncogenes, by chemical or physical carcinogens, or by infection with a retrovirus vector encoding the human telomerase catalytic subunit, hTERT cDNA. These cells were grown in Eagle's minimum essential medium containing $10 \%$ fetal bovine serum, $0.2 \mathrm{mM}$ serine, $0.1 \mathrm{mM}$ aspartic acid, $1.0 \mathrm{mM}$ pyruvate, and $0.22 \% \mathrm{NaHCO}_{3}$ at $37^{\circ}$ in a $5 \% \mathrm{CO}_{2}$ incubator.
DNA and RNA isolation. Genomic DNA was extracted from cells with a DNA extract kit (DNA Extractor WB kit, Wako Pure Chemical, Osaka, Japan). Total cellular RNAs were isolated from cells using the RNeasy mini kit (Qiagen, Hiden, Germany). Total RNAs $(2 \mu \mathrm{l})$ were reverse-transcribed with oligo(dT) primers by using the ready-to-go you-prime FirstStrand Beads (Amersham Bioscience, Piscataway, NJ) for first-strand cDNA synthesis according to the manufacturer's instructions.

DNA detection and RNA expression. Polymerase chain reaction $(\mathrm{PCR})$ and reverse transcriptase polymerase chain reaction (RT-PCR) were performed in a reaction mixture (25 $\mu \mathrm{l}$ ) containing $1 \mu \mathrm{l}$ of DNA (5 $\mathrm{ng}$ ) or RT reaction products (2.5 ng), 0.025 units of Taq polymerase (Ampli Taq Gold, Applied Biosystems, Foster City, CA), $200 \mu \mathrm{M}$ dNTP, $15 \mathrm{mM}$ Tris- $\mathrm{HCl}$ (pH 8.0), $50 \mathrm{mM} \mathrm{KCl,} 2.5 \mathrm{mM} \mathrm{MgCl}$, and $20 \mathrm{pmol}$ of forward and reverse primers (Table II) (13). The PCR products were subjected to a $2 \%$ agarose gel electrophoresis. The gels were stained with SYBR-Green I (Biowhittaker Molecular Applications, Rockland, ME) and analyzed by a fluorescence imaging analyzer (Luminescent image analyzer, LAS-1000 plus, Fuji film, Tokyo, Japan). To determine DNA polymorphisms within the transcribed region, imprinted genes amplified by PCR were digested with restriction enzymes and subjected to a $2 \%$ agarose gel electrophoresis. Human colorectal cancer cell lines (HTB-38 and CCL-247 cells) were used as the control cells with DNA polymorphism. In the present study, we assayed expressions of nine imprinted genes in the immortal cell lines 


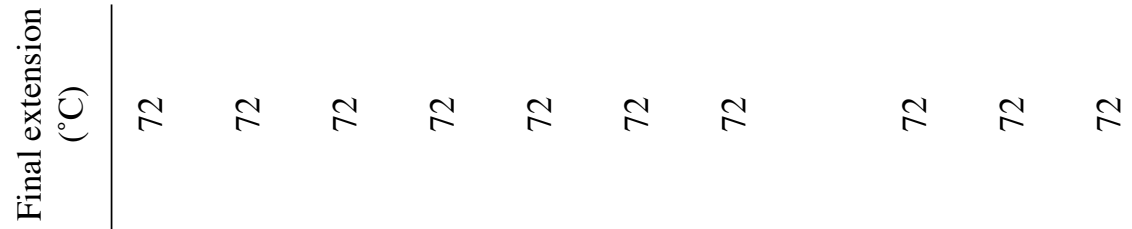

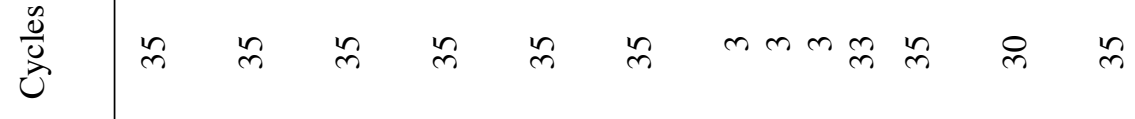

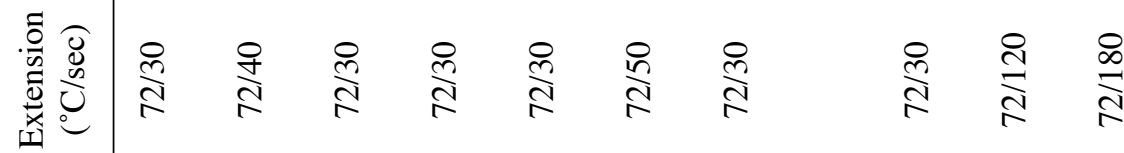

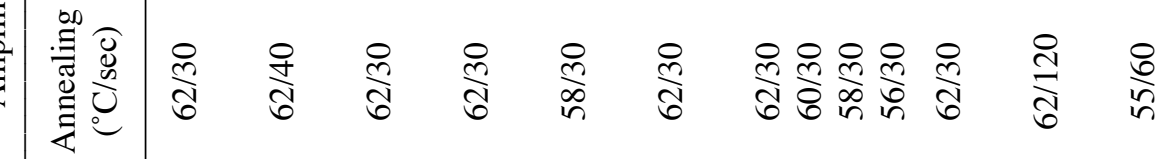

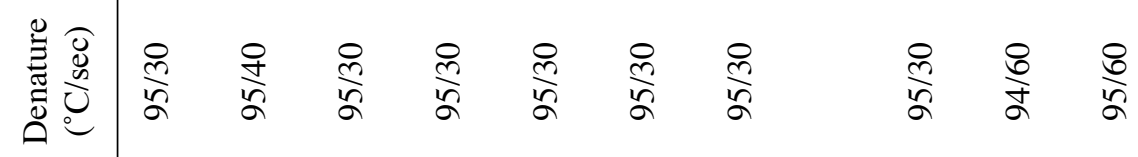

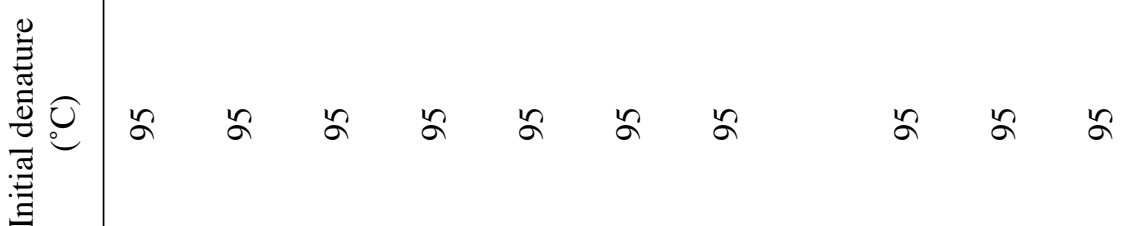

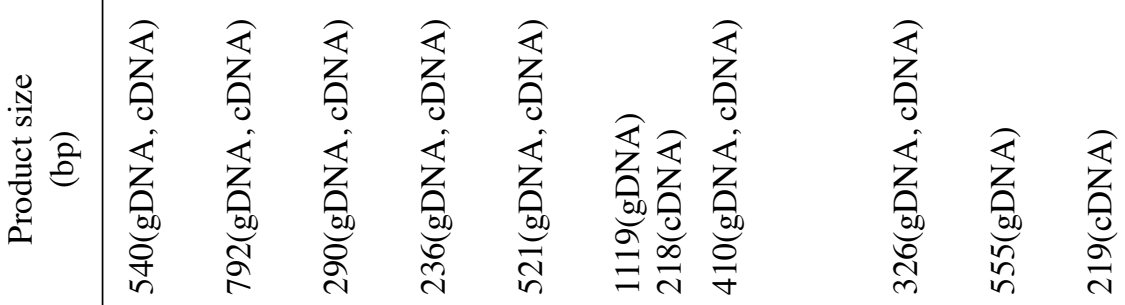

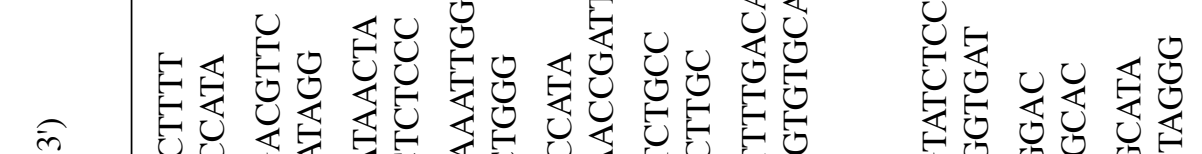

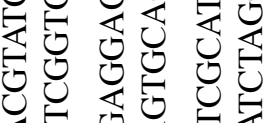

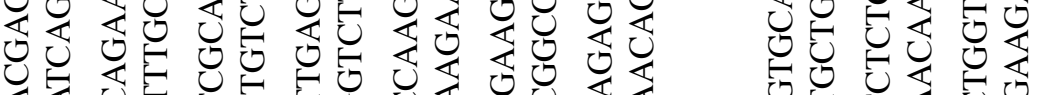

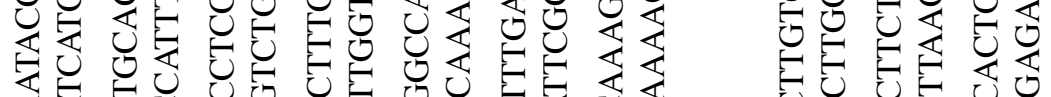

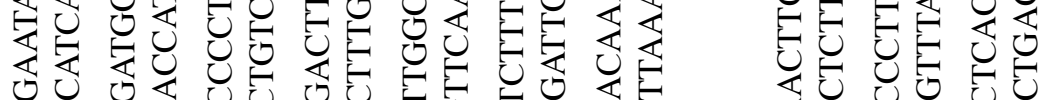

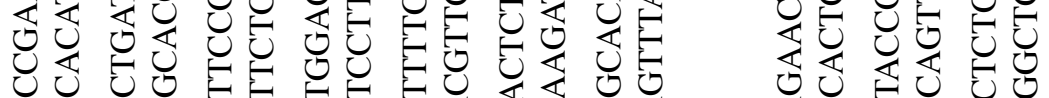

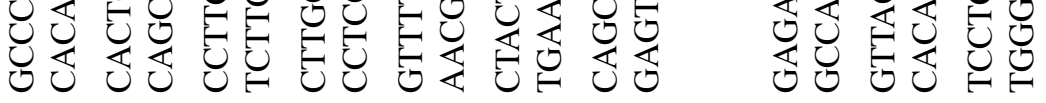


Table III. Expression of imprinted genes in the parental cells.

\begin{tabular}{cccccccccc}
\hline Cells & NDN & PEG1 & PEG3 & IGF2 & MAGE-L2 & SNRPN & LIT1 & NOEY2 & ZNF127 \\
\hline WHE-7 & + & + & + & + & + & + & + & + & + \\
\hline MDAH 087 & + & + & + & + & + & + & + & + & - \\
\hline WI-38 & + & + & + & + & + & + & + & + & - \\
\hline OUSM-24 & + & + & + & + & + & + & + & + & + \\
\hline KMS-6 & + & + & + & + & + & + & + & + & + \\
\hline Ay & + & + & + & + & + & + & + & + & + \\
\hline Pel & + & + & + & + & + & + & + & + & - \\
\hline
\end{tabular}

, polymorphic by restriction digest; $\square$, not polymorphic; , not determined; + or - , expression positive or negative, respectively.

by RT-PCR analysis and compared with those in their seven parental cells. We measured loss of expression of the imprinted genes. We also scored gain of expression of the polymorphic allele of the imprinted genes, including re-expression of non-expressed genes and biallelic expression of normally monoallelic expressed genes. We referred to both as LOI. When monoallelic expression of imprinted genes was maintained, we scored this as conserved. For imprinted genes with conserved expression in cells without DNA polymorphisms, we scored the results as not clear, because we did not distinguish whether the expression was monoallelic or biallelic. Because we failed to determine whether the expression of an imprinted gene, ZNF127, was monoallelic or biallelic, changes in the expression of the gene in the immortal cell lines were scored as follows. When the expression was positive in the parental cells but negative in the immortal cell lines, we scored this as loss of expression. When the expression was negative in the parental cells but positive in the immortal cell lines, we scored this as gain of expression. When the expression was positive in both the parental and immortal cells, we scored as not clear. When the expression was negative in both the parental and immortal cells, we scored as conserved.

Chromatin immunoprecipitation (ChIP) assay. The ChIP assay was performed with a ChIP assay kit (Upstate Biotechnology, Lake Placid, NY) with anti-dimethyl-histone H3 (Lys 9) and anti-dimethyl-histone H3 (Lys 4), as described previously $(13,14)$. These antibodies were purchased from Upstate Biotechnology. Immunoprecipitated DNA was amplified with an advantage-GC genomic polymerase mix (Clontech, Palo Alto, CA). The primers used for amplification of the promoter region of $N D N$ which is from $190 \mathrm{bp}$ upstream to $408 \mathrm{bp}$ downstream of the transcription site and the PCR conditions are shown in Table II. CEN16 was used as an internal control.
The primer sequences of CEN16 and the PCR conditions were the same as those described by Xin et al (15). The PCR products were subjected to a $2 \%$ agarose gel electrophoresis. The gels were stained and analyzed as described above.

Treatment of cells with 5-aza-2'-deoxycytidine (5-AZC). Cells were treated with $3 \mu \mathrm{M}$ 5-AZC (Sigma-Aldrich, Tokyo, Japan) for $96 \mathrm{~h}$.

\section{Results}

DNA polymorphisms of imprinted genes in the parental cells. Gel electrophoresis patterns representing DNA polymorphisms of imprinted genes within the transcribed region in the seven parental cells are shown in Fig. 1A. When compared with HTB-38 or CCL-247 cells used as the control cells with DNA polymorphisms, WHE-7 cells displayed DNA polymorphisms in $N D N, I G F 2$ and $S N R P N$. DNA polymorphism of imprinted genes in the other parental cells was also analyzed and the results are shown in Table III. All the parental cells expressed the nine imprinted genes with the exception of ZNF127, which was not expressed in three of the seven parental cells.

Changes in expression of imprinted genes with DNA polymorphisms. Typical examples of changes in imprinted gene expression in immortal cells and their parental WHE-7 cells with DNA polymorphisms within the transcribed region are shown in Fig. 1B. WHE-7 cells expressed NDN, IGF2 and $S N R P N$. The cDNA products of the parental WHE-7 cells which were digested with $N d e \mathrm{II}, A p a \mathrm{I}$, or Bst $U \mathrm{I}$, respectively, had a single band, indicating monoallelic expression of the imprinted genes. $N D N$ expressions were lost in seven of the eight immortal cell lines. $N D N$ expression was conserved 
A

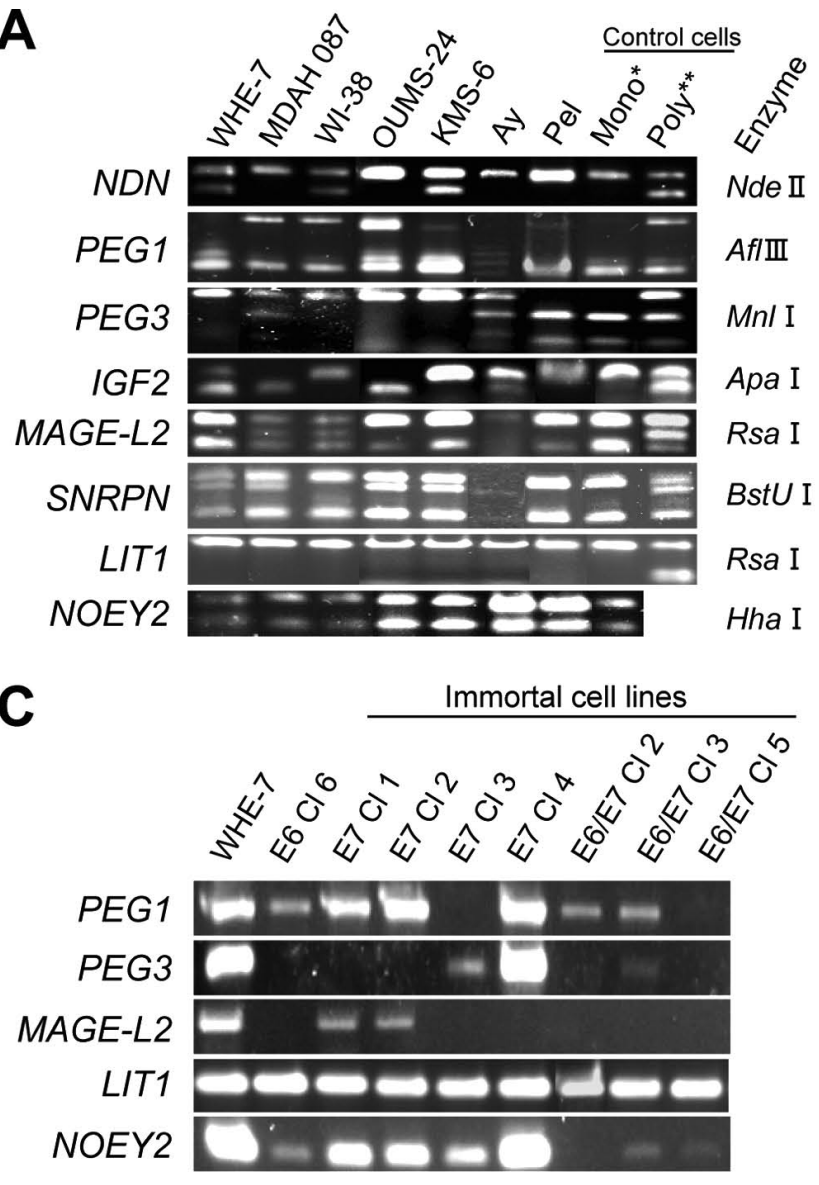

B

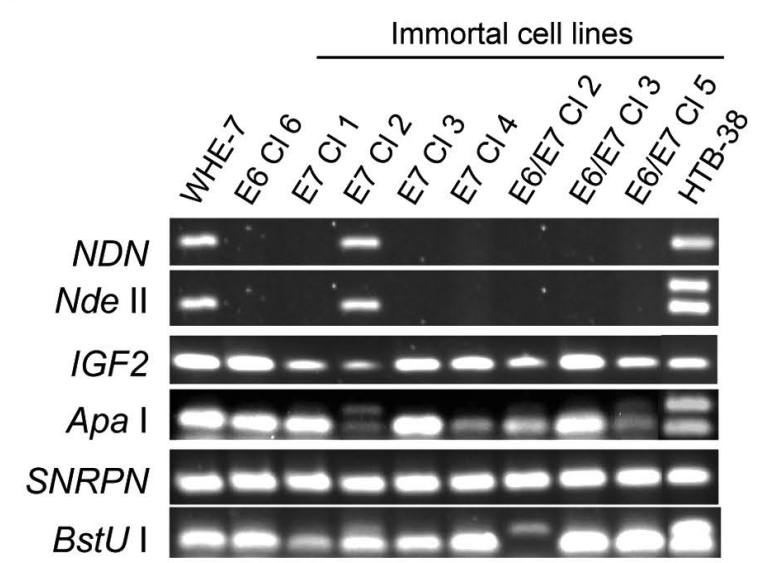

D

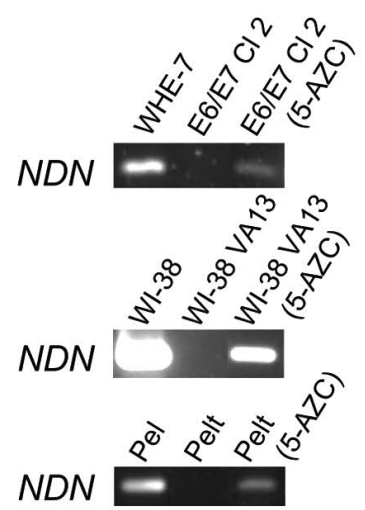

Figure 1. Expression of imprinted genes in human fibroblasts. (A) DNA polymorphism of imprinted genes in the parental cells. Following amplification by PCR, imprinted genes were digested with the indicated restriction enzymes and subjected to a $2 \%$ agarose gel electrophoresis. *Control cells with monomorphism; **Control cells with polymorphism. HTB-38 or CCL-247 cells were used for the control cells. (B) Loss and gain of expression of polymorphic, imprinted genes in human immortal cell lines derived from WHE-7 cells. HTB-38 cells were used as a positive control of cells with biallelic expression. After RT-PCR, cDNA of imprinted genes was digested with the indicated restriction enzymes and subjected to a $2 \%$ agarose gel electrophoresis. HTB-38 cells were used as control cells with biallelic expression. (C) Loss of expression of monomorphic, imprinted genes in human immortal cell lines derived from WHE-7 cells. (D) Restoration of $N D N$ expression in human immortal cell lines by 5-aza-2'-deoxycytidine (5-AZC).

in E7Cl 2 cells. IGF2 expressions were observed in all the eight immortal cell lines. However, the cDNA products in E7Cl 2 and E6/E7Cl 5 cells which were digested with ApaI had two bands, indicating biallelic expression of the imprinted gene. SNRPN expressions were also observed in all the eight immortal cell lines. The cDNA product in E6/E7Cl 2 cells which was digested with BstUI had a single band, but the position of band was shifted. We did not know what this meant. So, we scored this as not clear in this case. The cDNA products in the other seven cell lines which were digested with $B s t U \mathrm{I}$ had a single band, indicating that the gene expressions were conserved.

Changes in expression of imprinted genes without DNA polymorphisms. The parental WHE-7 cells did not display DNA polymorphisms in PEG1, PEG3, MAGEL-2, LIT 1 and NOEY2 (Fig. 1A). As shown in Fig. 1C, all the imprinted genes were expressed in WHE-7 cells, but some imprinted genes were not expressed in the immortal cell lines. The expression of the imprinted genes was lost in $\mathrm{E} 7 \mathrm{Cl} 3$ and E6/E7Cl 5 for PEGl, $\mathrm{E} 6 \mathrm{Cl} 6, \mathrm{E} 7 \mathrm{Cl} 1, \mathrm{E} 7 \mathrm{Cl} 2, \mathrm{E} 6 / \mathrm{E} 7 \mathrm{Cl} 2$, and E6/E7Cl 5 for $P E G 3$, all the immortal cell lines except for E7Cl 1 and E7Cl 2 for MAGE-L2, and E6/E7Cl 2 for NOEY2. Loss of LIT1 expres- sion was not observed in any of the immortal cell lines. The expression of each imprinted gene was conserved in the other immortal cell lines.

LOI in the immortal cell lines. All the results obtained from RT-PCR analysis are summarized in Table IV. LOI was observed in all the immortal cell lines that we examined. Genes most commonly exhibiting LOI were NDN, PEG3, MALE-L2 and ZNF127 which showed LOI in 15, 9, 11 and 9 of the 18 cell lines, i.e. $83 \%$ for $N D N, 61 \%$ for $M A G E-L 2$, and $50 \%$ for PEG3 and ZNF127.

Involvement of DNA methylation in LOI. Next, we examined an involvement of DNA methylation in LOI in the immortal cell lines. As shown in Fig. 1D, loss of $N D N$ expressions in the immortal cell lines was restored by treatment with an inhibitor of cytosine methyltransferase, 5-AZC.

ChIP assay in the promoter region of NDN. Because a difference in the methylation status was found between the parental cells and their immortal cell lines, ChIP assay of methylation patterns of histone $\mathrm{H} 3$ lysine 9 (H3-K9) and histone H3 lysine $4(\mathrm{H} 3-\mathrm{K} 4)$ in order to examine a possible involvement 
Table IV. Expression of imprinted genes in human immortal cell lines.

\begin{tabular}{|c|c|c|c|c|c|c|c|c|c|c|}
\hline \multirow{2}{*}{$\begin{array}{l}\text { Parental } \\
\text { cells }\end{array}$} & \multirow{2}{*}{$\begin{array}{l}\text { Immortal } \\
\text { cell lines }\end{array}$} & \multicolumn{9}{|c|}{ Imprinted genes } \\
\hline & & $N D N$ & PEG1 & PEG3 & IGF2 & MAGE-L2 & SNRPN & LIT1 & NOEY2 & ZNF127 \\
\hline \multirow{8}{*}{ WHE-7 } & $\mathrm{E} 6 \mathrm{Cl} 6$ & & & & & & & & & \\
\hline & $\mathrm{E} 7 \mathrm{Cl} 1$ & & & & & & & & & \\
\hline & $\mathrm{E} 7 \mathrm{Cl} 2$ & & & & & & & & & \\
\hline & $\mathrm{E} 7 \mathrm{Cl} 3$ & & & & & & & & & \\
\hline & $\mathrm{E} 7 \mathrm{Cl} 4$ & & & & & & & & & \\
\hline & E6/E7CI 2 & & & & & & & & & \\
\hline & E6/E7Cl 3 & & & & & & & & & \\
\hline & E6/E7Cl 5 & & & & & & & & & \\
\hline \multirow{5}{*}{ MDAH 087} & LCS-ST & & & & & & & & & \\
\hline & LCS-AF.1-2 & $*$ & & & & & $*$ & & & \\
\hline & LCS-AF.1-3 & & & & & & & & & \\
\hline & LCS-AF.3-1 & & & & & & & & & \\
\hline & LCS-4X2 & & & & & & & & & \\
\hline WI-38 & WI-38 VA13 & & & & & & & & & \\
\hline OUSM-24 & OUMS-24F & & & $*$ & & & & & & \\
\hline KMS-6 & KMST-6 & & & & & & & & & \\
\hline Ay & Ayt & & & & & & & & & \\
\hline Pel & Pelt & & & & & & & & & \\
\hline
\end{tabular}

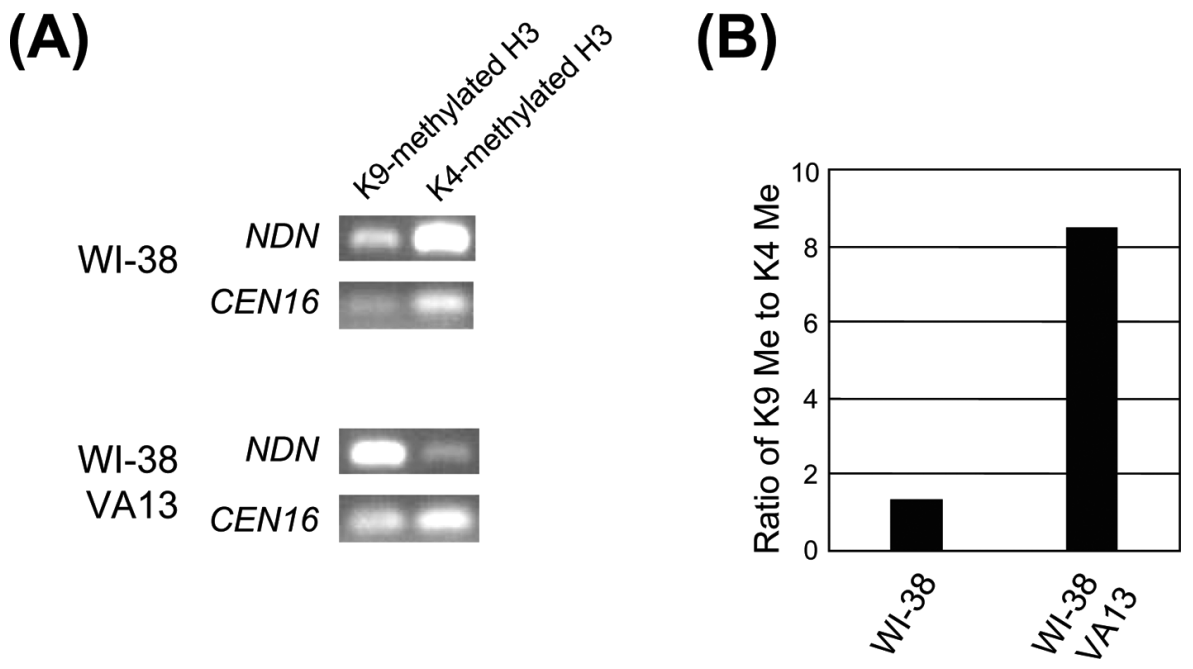

Figure 2. Methylation of histone H3. (A) Methylation status of histone H3 lysine 9 (H3-K9) and histone H3 lysine 4 (H3-K4) detected by ChIP assay in the promoter region of $N D N$ in WI-38 cells and their human immortal cell line, WI-38 VA 13 cells. CEN16 was used as the internal control. ChIP PCR products were subjected to a $2 \%$ agarose gel electrophoresis. The gels were stained with SYBR-Green I, the UV signals of which were quantified by densitometry with Software LabWorks version 4.0 (UVP, Upland, CA, USA). (B) Ratio of histone H3 lysine (K9) methylation (K9 Me) to histone H3 lysine 4 (K4) methylation (K4 Me) in WI-38 cells and their human immortal cell line, WI-38 VA13 cells.

of chromatin states in the transcriptional regulation of $N D N$ promoter was performed. Methylation of $\mathrm{H} 3-\mathrm{K} 4$ is well known to be associated with an open chromatin configuration such as that found at transcriptionally active promoter. In contrast, methylation of H3-K9 is a marker of condensed, inactive chromatin (16). The NDN promoter in the parental WI-38 cells exhibited hypomethylation of $\mathrm{H} 3-\mathrm{K} 9$ and hypermethylation of H3-K4 (Fig. 2). Conversely, the $N D N$ promoter in the immortal WI-38 VA 13 cells exhibited hypermethylation of H3-K9 and hypomethylation of H3-K4. The ratio of H3-K9 to $\mathrm{H} 3-\mathrm{K} 4$ methylation was much greater in the immortal cell line than in the parental cells. 


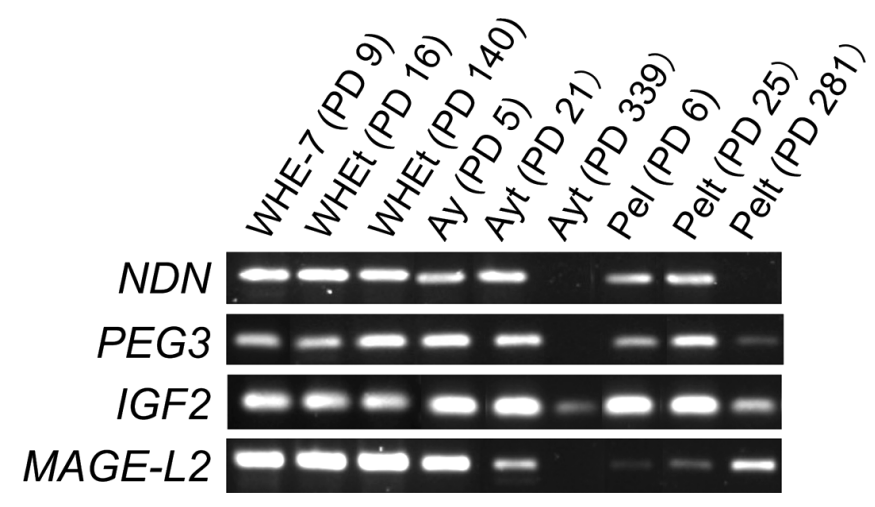

Figure 3. Expression of imprinted genes in human fibroblasts with an extended lifespan and in human immortal cell lines. WHEt cells (PD 140) are human fibroblasts with an extended lifespan that eventually senesced. Ayt cells (PD 339) and Pelt cells (PD 281) are human immortal cell lines. WHEt, Ayt and Pelt cells were derived from their parental cells, WHE-7, Ay and Pel cells, respectively, which were infected with a retrovirus vector encoding human telomerase catalytic subunit ( $h T E R T)$ cDNA (12).

Expression of imprinted genes in human fibroblasts with an extended lifespan that eventually senesce. We previously demonstrated that inactivation of the $\mathrm{p} 16^{\mathrm{INK} 4 \mathrm{a}} / \mathrm{pRb}$ pathway is necessary for immortalization of human fibroblasts (12). To examine the correlation between the inactivation of this pathway and LOI, we studied expression of imprinted genes in the human telomerase catalytic subunit hTERT cDNA-transduced human fibroblasts, i.e. WHEt, Ayt and Pelt cells. Each of their parental cells, i.e. WHE-7, Ay and Pel cells senesced at 70 (8), 10 (12) and 12 (12), respectively. WHEt cells had a considerable extension of lifespan, but eventually senesced at 207 population doublings (PD). Conversely, Ayt and Pelt cells were immortal because they maintained a vigorous growth for over 400 PD (12). WHEt cells at PD 20 and PD 190 expressed p16 protein and mRNA and the CpG islands within the promoter region of INK $4 a$ were unmethylated. Conversely, immortal Ayt and Pelt cells failed to express p16 protein and mRNA. Similar levels of two signals showing methylation and unmethylation of the INK4a CpG islands were observed in the immortal Ayt and Pelt cells. Both cells had a hyperphosphorylated $\mathrm{Rb}$ protein (12).

As shown in Fig. 3, the expression of $N D N$ and IGF2 was conserved in WHEt cells (PD 140). Conversely, the expression of NDN, PEG3 and MAGE-L2 was lost in the immortal Ayt cells (PD 339) and the expression of NDN was lost in the immortal Pelt cells (PD 281). In this connection, these gene expressions were conserved in Ayt and Pelt cells in the precrisis phase. Altogether, human fibroblasts in the pre-crisis phase and cells with an extended lifespan that eventually senesce, both of which have the normal $\mathrm{p} 16^{\mathrm{INK} 4 \mathrm{a}} / \mathrm{pRb}$ pathway, did not show LOI at any imprinted gene examined.

\section{Discussion}

LOI of nine imprinted genes in 18 human immortal fibroblast cell lines was examined. LOI was observed in all the immortal cell lines. Cells with an extended lifespan that eventually senesce as well as cells in the pre-crisis phase did not show LOI. The gene most commonly exhibiting LOI was NDN.
The other genes exhibiting LOI at high frequencies were MAGE-L2, PEG3 and ZNF127. NDN, MAGE-L2 and ZNF127 are mapped to the human chromosome 15q11-q13. Their expressions are critical for Prader-Willi syndrome (PWS) which is a neurodevelopmental disorder. The genes are maternally imprinted and transcribed only from the paternal allele (17). NDN which codes the nuclear-binding protein necdin is mapped to human chromosome 15q11.2-q12, a region deleted in PWS (17-19). Necdin appears in most of the terminally differentiated neurons in the central and peripheral nervous systems and suppresses the cell growth and apoptosis $(18,20)$. Necdin binds to Simian virus 40 (SV 40) large T antigen, adenovirus E1A, and the transcription factor E2F1 and acts as a growth suppressor, like $\mathrm{pRb}$ (21). Necdin also interacts with the tumor suppressor gene product p53 and inhibits p53-mediated apoptosis (22). These findings suggest that necdin blocks cell cycle reentry of postmitotic neurons and promotes their survivals by preventing p53-mediated apoptosis.

The NDN gene product necdin acts in many human tissues besides neurons. Necdin in combination with Msx 2, a transcription factor, plays a crucial role in both the smooth muscle differentiation and the maintenance of smooth muscles in the differentiated state (23). NDN is expressed in normal human fibroblasts derived from skin, gingiva and periodontal ligament as shown in the present study. Expression of $N D N$ is downregulated in human melanoma cells (24), mouse neuroblastoma cells (25), NIH3T3 cells (20) and monkey kidney cells transformed by SV 40 (21). Ectopic expression of necdin induces a growth arrest in human melanoma cells (24) and NIH3T3 cells (20) as well as results in a growth arrest and a restoration of neuronal phenotype in mouse neuroblastoma cells (25).

Imprinting in the chromosome 15q11-q13 region, including NDN, MAGE-L2, ZNF127 and SNRPN which are paternally expressed genes, is under the control of an imprinting center (IC) located in the 5' region of SNRPN (26). The mouse homologues of these genes map to mouse chromosome 7 in a region syntenic with human chromosome 15q11-q13 (17). Mice heterozygous for the paternally inherited IC-deletion die as neonates due to methylation of the paternal allele of the genes (27).

Because (I) DNA methylation is a common mechanism of genomic imprinting and (II) LOI of SNRPN was rare in the immortal cell lines when compared with that of the other imprinted genes under the control of the same IC, such as NDN, $M A G E-L 2$ and ZNF127, we considered that DNA methylation was one of the possible mechanisms of LOI in the imprinted genes that we examined. Loss of $N D N$ expression in the immortal cell lines was restored by 5-AZC. The result suggests that an epigenetic mechanism related to DNA methylation is participating in the LOI of NDN and that a modification of chromatin state may be involved in the transcriptional regulation of $N D N$ promoter. Our findings obtained from ChIP assay indicate that the chromatin containing the $N D N$ promoter is heterochromatic in the immoral cell line, suggesting that the reversible conversion of $N D N$ expression between the parental cells and their immortal cell lines is attributed to the epigenetic mechanism by chromatin structure-mediated regulation of $N D N$ expression. A change in the expression of imprinted gene by chromatin modification was also observed in the other 
imprinted gene. Shibui et al (13) in our laboratory demonstrated that changes by sodium arsenite in the expression of SNRPN in human cells can be associated with the chromatin structuremediated regulation of SNRPN promoter.

Reynolds et al (28) have demonstrated that upon removal of p16 ${ }^{\mathrm{INK} 4 \mathrm{a}}$ activity in primary human mammary epithelial cells, polycomb repressors, EZH2 and SUZ12, are upregulated and recruited to $H O X A 9$, a locus expressed during normal breast development and epigenetically silenced in breast cancer. The upregulation of polycomb repressors leads to the recruitment of DNA methyltransferases and the downregulation of expression of the targeted locus, HOXA9, by DNA hypermethylation (28). These findings suggest the important role of DNA hypermethylation by the recruitment of DNA methyltransferase caused by inactivation of $\mathrm{p} 16^{\mathrm{INK} 4 \mathrm{a}}$ and $\mathrm{pRb}$ pathway in immortalization of human cells. LOI was detectable in the immortal cell lines but undetectable in their cells either in the pre-crisis phase or with an extended lifespan that eventually senesce, meaning that LOI can have a close correlation with immortalization. Because the inactivation of p16 $6^{\mathrm{INK} 4 \mathrm{a}}$ and $\mathrm{pRb}$ pathway is associated with immortalization of human cells, including fibroblasts, as described previously (12), it is very interesting to clarify the correlation between LOI and the recruitment of DNA methyltransferase in the immortal cell lines that we used in the present study.

The human cell lines used were derived from human fibroblasts which had been immortalized spontaneously, by viral oncogenes, by chemical or physical carcinogens, or by infection with a retrovirus vector encoding hTERT cDNA. LOI was observed in all the cell lines independent of the factors by which human fibroblasts became immortal. In addition, Shibui et al (13) have demonstrated that treatment of human cells with various mutagenic or non-mutagenic carcinogens, e.g. mitomycin C, 4-nitroquinoline 1-oxide, diethylstilbestrol, sodium arsenite and nickel chloride, induces LOI independent of their mutagenic activity. These suggest that epigenetic modifications act as an independent factor necessary for the conversion of normal human cells into cells with immortality. Holm et al (4) suggest the possibility that LOI at key loci encoding tumor suppressors and oncogenes provides the first step toward tumor formation by conferring cellular immortality.

In the present study, to investigate the possible involvement of LOI in immortalization of human fibroblasts, expressions of nine imprinted genes in 18 human immortal fibroblast cell lines were compared with those in their seven parental cells. We demonstrated that immortalization of human fibroblasts was accompanied by LOI. Although it is not clear if LOI plays a causal role in immortalization of human cells or is merely coincidental, the finding indicates a possible involvement of LOI in immortalization of human fibroblasts or a common mechanism involved in both processes.

\section{References}

1. Li Y, Meng G and Guo Q-N: Changes in genomic imprinting and gene expression associated with transformation in a model of human osteosarcoma. Exp Mol Pathol 84: 234-239, 2008.

2. Reik W and Walter J: Genomic imprinting: parental influence on the genome. Nat Rev Genet 2: 21-32, 2001.

3. Robertson KD: DNA methylation and human disease. Nat Rev Genet 6: 597-610, 2005.
4. Holm T M, Jackson-Grusby L, Brambrink T, et al: Global loss of imprinting leads to widespread tumorigenesis in adult mice. Cancer Cell 8: 275-285, 2005.

5. Yamamoto A, Kumakura S, Uchida M, et al: Immortalization of normal human embryonic fibroblasts by introduction of either the human papillomavirus type 16 E6 or E7 gene alone. Int J Cancer 106: 301-309, 2003.

6. Bischoff F Z, Yim S O, Pathak S, et al: Spontaneous abnormalities in normal fibroblasts from patients with Li-Fraumeni cancer syndrome: aneuploidy and immortalization. Cancer Res 50: 7979-7984, 1990.

7. Tsutsui T, Fujino T, Kodama S, et al: Aflatoxin $\mathrm{B}_{1}$-induced immortalization of cultured skin fibroblasts from a patient with Li-Fraumeni syndrome. Carcinogenesis 16: 25-34, 1995.

8. Tsutsui T, Tanaka Y, Matsudo Y, et al: Extended lifespan and immortalization of human fibroblasts induced by X-ray irradiation. Mol Carcinog 18: 7-18, 1997.

9. Girardi A J, Weinstein D and Moorhead PS: SV 40 transformation of human diploid cells. A parallel study of viral and karyologic parameters. Ann Med Exp Biol Fenn 44: 242-254, 1966.

10. Bai L, Mihara K, Kondo Y, et al: Immortalization of normal human fibroblasts by treatment with 4-nitroquinoline 1-oxide. Int J Cancer 53: 451-456, 1993.

11. Namba M, Nishitani K, Hyodoh F, et al: Neoplastic transformation of human diploid fibroblasts (KMST-6) by treatment with ${ }^{60}$ Co gamma rays. Int J Cancer 35: 275-280, 1985.

12. Tsutsui T, Kumakura S, Yamamoto A, et al: Association of p16 $6^{\mathrm{NK} 4 \mathrm{a}}$ and $\mathrm{pRb}$ inactivation with immortalization of human cells. Carcinogenesis 23: 2111-2117, 2002.

13. Shibui T, Higo Y, Tsutsui TW, et al: Changes in expression of imprinted genes following treatment of human cancer cell lines with non-mutagenic or mutagenic carcinogens. Int J Oncol 33: 351-360, 2008.

14. Kumakura S, Tsutsui TW, Yagisawa J, et al: Reversible conversion of immortal human cells from telomerase-positive to telomerase-negative cells. Cancer Res 65: 2778-2786, 2005.

15. Xin Z, Allis C D and Wagstaff J: Parent-specific complementary patterns of histone $\mathrm{H} 3$ lysine 9 and $\mathrm{H} 3$ lysine 4 methylation at the Prader-Willi syndrome imprinting center. Am J Hum Genet 69: 1389-1394, 2001.

16. Peters A H, Mermoud J E, O'Carroll D, et al: Histone H3 lysine 9 methylation is an epigenetic imprint of facultative heterochromatin. Nat Genet 30: 77-80, 2002.

17. MacDonald H R and Wevrick R: The necdin gene is deleted in Prader-Willi syndrome and is imprinted in human and mouse. Human Mol Genet 6: 1873-1878, 1997.

18. Uetsuki T, Takagi K, Sugiura H, et al: Structure and expression of the mouse necdin gene. Identification of a postmitotic neuronrestrictive core promoter. J Biol Chem 271: 918-924, 1996.

19. Nakada Y, Tanimura H, Uetsuki T, et al: The human chromosomal gene for necdin, a neuronal growth suppressor, in the Prader-Willi syndrome deletion region. Gene 213: 65-72, 1998.

20. Hayashi Y, Matsuyama K, Takagi K, et al: Arrest of cell growth by necdin, a nuclear protein expressed in postmitotic neurons. Biochem Biophys Res Commun 213: 317-324, 1995.

21. Tanimura H, Taniguchi N, Hara M, et al: Necdin, a postmitotic neuron-specific growth suppressor, interacts with vital transforming proteins and cellular transcription factor E2F1. J Bio Chem 273: 720-728, 1998.

22. Tanimura H, Matsumoto K and Yoshikawa K: Physical and functional interactions of neuronal growth suppressor necdin with p53. J Biol Chem 274: 16242-16248, 1999.

23. Brunelli S, Tagliafico E, DeAngelis FG, et al: Msx 2 and necdin combined activities are required for smooth muscle differentiation in mesoangioblast stem cells. Circ Res 94: 1571-1578, 2004.

24. Hoek K, Rimm DL, Williams KR, et al: Expression profiling reveals novel pathways in the transformation of melanocytes to melanomas. Cancer Res 64: 5270-5282, 2004.

25. Kobayashi M, Tanimura $\mathrm{H}$ and Yoshikawa K: Ectopic expression of necdin induces differentiation of mouse neuroblastoma. $\mathrm{J}$ Biol Chem 277: 42128-42135, 2002.

26. Horsthemke B: Structure and function of the human chromosome 15 imprinting center. J Cell Physiol 173: 237-241, 1997.

27. Yang T, Adamson TE, Resnick JL, et al: A mouse model for Prader-Willi syndrome imprinting-centre mutations. Nat Genet 19: 25-31, 1998.

28. Reynolds PA, Sigaroudinia M, Zardo G, et al: Tumor suppressor p16 ${ }^{\text {INK4a }}$ regulates polycomb-mediated DNA hypermethylation in human mammary epithelial cells. J Biol Chem 281: 24790-24802, 2006. 